\title{
Malnutrición materno-fetal: Revisión de la bibliografía internacional y la urgencia de estudios, prevención e intervención en el Perú
}

\author{
Maternal-fetal malnutrition: Literature review and the urgent call for conducting more studies in Peru \\ Jorge E. Uceda ${ }^{1,2}$, Luís Caravedo-Reyes ${ }^{3, a}$, Maria Luísa Figueroa ${ }^{4, c}$ \\ RESUMEN \\ En el Perú, la malnutrición materno-fetal ocurre con frecuencia, pero su prevención, intervención y seguimiento \\ no ha sido investigada en relación al conocimiento y experiencia de países emergentes. Los autores presentan una \\ revisión actualizada de los avances científicos sobre malnutrición materno-fetal, con el fin de prevenir y manejar un \\ empobrecido capital humano, que constituye nuestro principal y más urgente problema sanitario.
}

PALABRAS CLAVE: Desnutrición, recursos humanos, prevención de enfermedades. (Fuente: DeCS BIREME).

\section{SUMMARY}

Maternal-fetal malnutrition is common in Peru, but its prevention and possible intervention measures have not been investigated in Peru. We conducted a literature review on the topic to prevent and manage this serious public health problem.

KEYWORDS: Malnutrition, workforce, disease prevention. (Source: MeSH NLM).

\section{INTRODUCCIÓN}

Por décadas, hemos observado niños peruanos que crecen en pobreza y sus consecuencias son evidentes: desnutrición, anemia, subdesarrollo pondoestatural y marcada deficiencia para el aprendizaje. Las intervenciones sanitarias han sido muy variables e incompletas, conformando un crónico y poco estudiado problema de salud pública. Este desolador panorama se iniciaría con la malnutrición de la gestante, produciéndose alteraciones placentarias y una serie de defectos genéticos y neurológicos fetales, la mayoría de los cuales son perfectamente previsibles.

Por las razones expuestas consideramos necesario llevar a cabo una revisión bibliográfica de las principales revistas médicas internacionales sobre la malnutrición materno-fetal y así identificar información aplicable en el Perú.

\footnotetext{
American Board of General Surgery.

American Board of Pediatric Surgery.

Universidad Peruana Cayetano Heredia. Lima, Perú.

Consultora en Nutrición Materno Infantil.

Profesor Emérito.

Magister en Nutrición.
} 


\section{Primeras hipótesis}

En 1989, David Barker y colaboradores introdujeron la hipótesis "Orígenes fetales de la enfermedad en los adultos", este trabajo se realizó en la Universidad de Southampton, estudiaron adultos con diabetes tipo 2 y enfermedad cardíaca que habían nacido con bajo peso y propusieron que durante el período crítico del desarrollo prenatal, una deficiente nutrición materna podría haber alterado la anatomía y el metabolismo fetal seguido más tarde por enfermedades endocrinas y cardiovasculares ${ }^{(1)}$. Esta posible relación originó serias consideraciones sobre la salud pública de países en vías de desarrollo, cuyo porcentaje de neonatos de bajo peso es seis veces más frecuente que en países desarrollados ${ }^{(2)}$.

Con el advenimiento del siglo XXI el fenómeno de la "programación fetal" señalaba que la desnutrición materna actuaba sobre el genoma fetal originando alteraciones permanentes en la salud ${ }^{(3,4)}$.

En 2008, la revista Lancet publicó una serie de artículos sobre desnutrición materna e infantil, reconociendo su importancia como problema sanitario y la necesidad de un manejo precoz y el reconocimiento de sus efectos a largo plazo ${ }^{(5-9)}$.

\section{Alteraciones placentarias}

En 2010, Belkacemi y col. (10), confirmaron que la desnutrición materna afecta el desarrollo placento-fetal, produciendo retardo del crecimiento intrauterino (IUGR siglas del inglés), aumento en la morbimortalidad perinatal y neonatos de bajo peso que tienen un alto riesgo para desarrollar el síndrome metabólico del adulto. Las alteraciones placentarias varían con el entorno nutricional e incluyen disminución o aumento de tamaño, alteración del desarrollo vascular, expresión disminuida del factor de crecimiento angiogénico y un transporte reducido de glucosa, amino ácidos y lípidos. Los efectos sobre la expresión genética pueden programar futuras generaciones debido a efectos epigenéticos sobre el genoma placentario, el genoma fetal, o ambos ${ }^{(10)}$.

\section{Micronutrientes}

Diferentes estudios han demostrado la importancia del aporte de cinco oligoelementos: hierro, zinc, iodo, folato y vitamina A en el desarrollo del feto, durante la gestación o en la etapa pre-gestacional de la madre. En nuestro medio no existe un programa de suplementación de estos elementos y podemos asumir que la proporción de mujeres con deficiencias en estos cinco micronutrientes sería muy alta, ya que, en países desarrollados, un meta-análisis de 62 estudios y 108733 gestantes encontró que la concentración de estos micronutrientes estaba por debajo de las recomendaciones internacionales ${ }^{(11-13)}$.

\section{El desarrollo fetal como origen de la salud y la enfermedad}

La hipótesis denominada "Developmental Origins of Health and Disease" (DOHaD) ó "El Desarrollo fetal como Origen de la Salud y la Enfermedad", es un concepto reciente que en los 1990's se llamó "Origen Fetal de la Enfermedad Adulta". En los últimos diez años ha sido frecuentemente estudiada ${ }^{(14-16)}$.

Caroline Fall ${ }^{(14)}$, de la Unidad de Epidemiología de la Universidad de Southampton, en 2013 publicó un estudio sobre el seguimiento prolongado de la malnutrición fetal. En él se discute cómo el retardo del crecimiento intrauterino (IUGR), es seguido de una amplia gama de afecciones, tales como un "pobre capital humano" (baja estatura, pobre desarrollo cognitivo); mayor riesgo para contraer otras enfermedades (hipertensión arterial, intolerancia a la glucosa, e hipofunción pulmonar, inmunológica y renal); y enfermedad clínica (diabetes, cardiopatía coronaria, neuropatías y nefropatías crónicas).

Los neonatos con alto peso en casos de diabetes gestacional, se han asociado con obesidad, diabetes y un mayor riesgo de cáncer. Múltiples estudios en animales han demostrado que la desnutrición maternofetal reproduce los problemas de salud en la edad adulta, incluyendo estudios de los fenómenos epigenéticos, tales como la metilación del ADN y su tratamiento con ácido fólico. Finalmente, el seguimiento a largo plazo de neonatos que recibieron intervención nutricional fetal, sólo demostró una leve mejoría en el crecimiento, función vascular, concentración de lípidos, tolerancia a la glucosa y resistencia a la insulina ${ }^{(14)}$.

La hambruna registrada en China entre 1959 y 1962, fue la base de un estupendo estudio de la Universidad Jiaotong de Shangai, en el que se evaluaron los efectos sobre la población fetal e infantil y la aparición del síndrome metabólico (SM) en la vida adulta; luego de una minuciosa selección, Wang y col. (Departamentos de Endocrinología/Metabolismo y Bioestadística) ${ }^{(15)}$, estudiaron 6445 personas. El síndrome metabólico fue definido según criterios de la Federación Internacional 
de Diabetes del 2005: incremento de la circunferencia abdominal, aumento de triglicéridos, disminución de HDL, hipertensión arterial e historia de diabetes; los resultados indicaron que los fetos/infantes expuestos, experimentaron una significativa mayor incidencia del SM en mujeres, más no así en hombres. Además, se anota que estos cambios ocurrieron más allá de "los primeros 1000 días", hasta los 9 años ${ }^{(15)}$.

El tercer artículo sobre el desarrollo fetal como origen de la salud y la enfermedad (DOHaD), sugiere que las intervenciones necesarias a llevarse a cabo en los países de bajos a medianos ingresos, sean medidas a largo plazo y practicadas por equipos multidisciplinarios que incluyan reducción de la pobreza, inversión en nutrición y educación, promoción de lactancia materna y nutrición infantil, mejoría del agua y alcantarillado, reducción de tabaco $\mathrm{y}$ alcohol y empoderamiento de las mujeres ${ }^{(16)}$.

\section{Retardo del crecimiento intrauterino (IUGR)}

El IUGR es un neonato con huellas de malnutrición fetal y con un estudio Doppler que muestra flujo sanguíneo anormal. El pequeño para edad gestacional (SGA), es un neonato con peso $<10^{\circ}$ percentil para su edad gestacional, o al menos dos desviaciones estándar por debajo de la población normal. El IUGR significa compromiso intrauterino y el SGA refleja el potencial genético del infante. Ambos, especialmente el SGA, ocurren en países ricos, pero el IUGR es seis veces más frecuente en países en vías de desarrollo. Anualmente, en el mundo, nacen 30 millones de infantes con IUGR, $75 \%$ en Asia, $20 \%$ en África y 5\% en Latinoamérica.

El IUGR es un serio problema de salud pública, constituyendo una de las mayores causas de mortalidad y morbilidad perinatal. Además, el IUGR puede desencadenar enfermedades crónicas del adulto. La morbilidad precoz incluye asfixia, aspiración de meconio, hipertensión pulmonar persistente, hipotermia, hipoglicemia, policitemia, ictericia, pobre alimentación, enterocolitis necrotizante y sepsis. La morbilidad tardía consiste en alteraciones neurológicas, enfermedad cardiovascular y síndrome metabólico.

\section{Etiología}

Se pueden dividir en materna, fetal o placentaria.

a) Materna: Demográficas, medio ambiente, problemas obstétricos y ginecológicos. b) Fetal: Genéticas: trisomías 13, 18, 21; malformaciones e infecciones congénitas.

c) Placentarias: Bajo peso, anomalías vasculares, infecciones.

\section{Diagnóstico postnatal}

a) Examen clínico: Pequeño, arrugado, alerta, cabeza grande, piel seca, areolas hipoplásicas, abdomen escafoides y pequeño, cordón hipoplásico, manos, pies y uñas largas, genitales inmaduros.

b) Índices antropométricos: Ponderal, perímetro brazo, perímetro brazo-cabeza, El puntaje CAN (evaluación clínica nutricional) es considerado el mejor; evalúa la pérdida del tejido subcutáneo y masa muscular en nueve regiones del neonato (mejillas, cuello, mentón, brazos, piernas, glúteos, tórax, abdomen y cabello); el puntaje varía de 1 a 4, malnutrición/normal; un puntaje $<25$ es diagnóstico de malnutrición.

\section{Tipos de IUGR}

i. Simétrico: $20-30 \%$.

Disminución de peso, talla y cabeza. Ocurre en primer trimestre. El número de células está reducido. Causas genéticas, anomalías, infecciones. Índice ponderal bajo, $<2$; pobre pronóstico, alta mortalidad y morbilidad.

ii. Asimétrico: $70-80 \%$.

Reducción de peso y talla para proteger cerebro. Ocurre al final del segundo o tercer trimestre. El número de células es normal, tamaño reducido.

\section{Manejo postnatal}

Comienza por precisar si la noxa fue temprana o tardía, si el IUGR es simétrica o asimétrica y si los mecanismos son maternos, fetales o placentarios. Son complicaciones inmediatas la mortalidad neonatal, asfixia perinatal, insuficiencia pulmonar, déficits metabólicos, gastrointestinales, termoreguladores, hematológicos, insuficiencia renal e inmunodeficiencia. Las complicaciones a largo plazo implican pobre crecimiento físico, síndrome metabólico, endocrinopatías, insuficiencia renal, problemas respiratorios, óseos, visuales y auditivos.

Todas estas alteraciones son tratadas por la Neonatología. Sin embargo, las deficiencias 
neurológicas son especialmente graves para la desprotegida población pobre mundial ${ }^{(17,18)}$.

\section{Desnutrición materno-fetal y alteraciones en el desarrollo neurológico}

El estudio pionero de Lucas de $1998^{(19)}$, fue una intervención nutricional de cuatro semanas en 360 infantes con menos de 1851 gramos de peso al nacer, aleatorio y ciego, seguido por 7,5-8 años con pruebas de inteligencia por el método de Weschler. Se empleó fórmula pre término enriquecida $(24 \mathrm{cal} / \mathrm{oz}$ ) y estándartérmino (20 cal/oz), lo que constituyó el ensayo A, con 160 infantes; 264 madres aportaron lactancia materna y ellas fueron seleccionadas al azar para suplementar a los infantes con fórmulas pre término o término, lo que constituyó el ensayo B.

Este estudio tuvo como resultados:

1. En ambos ensayos, los alimentados con $24 \mathrm{cal} / \mathrm{oz}$ ganaron más peso que los que recibieron $20 \mathrm{cal} / \mathrm{oz}$.

2. Las mediciones IQ, verbal, funcional y total a 7,58 años fueron significativamente mejores con la dieta fortificada, con o sin leche materna.

3. Estas diferencias fueron aún más evidentes en el sexo masculino, particularmente en IQ verbal y el total.

4. En los niños con parálisis cerebral y alimentados con fórmula de 20 cal/onza, la gran mayoría registró un IQ verbal bajo $(<85)$. Lucas, Morley y Cole, demostraron que una breve pero temprana manipulación dietética en infantes de bajo peso tiene importantes beneficios en su ulterior desarrollo cognitivo ${ }^{(19)}$.

En un trabajo publicado en el 2009 por Isaacs, Morley y Lucas ${ }^{(20)}$, en el que las mediciones de IQ fueron a los 8 y 16 años de edad (20), los autores señalan que numerosos estudios en animales han demostrado que la desnutrición fetal durante el desarrollo del sistema nervioso, puede afectar el desarrollo y la función cerebral en forma permanente. Trasladar estos hallazgos a humanos es, sin embargo, difícil. Los estudios observacionales en los países en vías de desarrollo son potencialmente desvirtuados por factores socioeconómicos y medioambientales. El aislamiento de los efectos de la nutrición y la demostración de sus efectos causales requiere estudios experimentales que involucren el azar grupal, tal como se mostró en el artículo anterior. Esta vez, las intervenciones dietéticas consistieron en fórmula enriquecida en 49 pacientes y otra estándar administrada a 46 pacientes. El número de sujetos en este estudio fue limitado por la inclusión de neuroimágenes. Además, solo participaron prematuros menores a 30 semanas de edad gestacional y niños que a $\operatorname{los} 8$ años fueron encontrados neurológicamente normales. El desarrollo de los 95 sujetos tuvo lugar en Londres; este estudio tuvo como resultados:

1. A la edad de 8 años, la dieta enriquecida fue estadísticamente superior a la estándar en todos los IQ, verbal, funcional y total.

2. La VIQ (verbal) persistió superior hasta la adolescencia, siendo el índice de comprensión verbal el componente más importante. Los autores concluyen que un breve período de intervención dietética después del parto prematuro, principalmente entre 26 y 34 semanas de gestación, afectó el coeficiente intelectual a los 16 años. La dieta de nutrientes estándar se asoció con un VIQ más bajo, debido principalmente a diferencias en la comprensión verbal, el cual persistió después de controlar los factores sociales ${ }^{(20)}$.

En 2014 se publicó una revisión sobre el impacto de la nutrición en el desarrollo del cerebro y sus implicancias neuroprotectoras después del parto prematuro ${ }^{(21)}$. Se sabe que el crecimiento y maduración cerebral fetal tiene lugar durante el final del segundo trimestre y en el tercer trimestre de la gestación ${ }^{(21)}$. El cerebelo y la corteza cerebral crecen rápidamente durante la gestación, seguidos por la sustancia blanca. La lesión cerebral más frecuente se localiza en la sustancia blanca como una leucomalacia quística peri ventricular o hemorragia intraventricular. Se producen disrupciones de las conexiones córtico-talámicas, atrofia cortical, déficits axonales y alteraciones en la mielinización. En el prematuro, el cerebelo se afecta con frecuencia. Todo ello se manifiesta por deficiencias funcionales en el desarrollo del infante, a menos que se instituya una nutrición precoz. Aquí se citan los estudios británicos de Alan Lucas. El eje microbiotaintestino-cerebro ha cobrado reciente importancia con investigaciones en ratones. Los ratones libres de gérmenes intestinales manifestaron mayor tranquilidad que los controles. Los ratones con el comportamiento alterado mostraron alteración en la concentración de neurotransmisores y en la expresión de factores neurotróficos. Al restituir la flora microbiana intestinal, precozmente, el comportamiento y la neuroregulación se normalizaron, lo cual no sucedió con la reposición de la flora en la adultez de los ratones. Finalmente, el empleo de ciertos componentes nutricionales demostró un rol neuroprotector (glicina, probióticos, vitamina $\mathrm{E}$ y L-arginina) ${ }^{(21)}$. 
Una investigación sobre el IUGR fue realizada por investigadores de la Universidad Jiao Tong, en la provincia de Shaanxi, mayormente rural y pobre, localizada en el oeste de China ${ }^{(22)}$, publicada en 2016, fue la continuación de otra llevada a cabo por los autores en la misma población de 479000 habitantes entre 2002 y 2006 para investigar tres grupos de micronutrientes en gestantes, mediante un ensayo doble ciego, con grupos aleatorios. Entre octubre de 2012 y setiembre de 2013, se entrevistaron a 1744 niños que permanecieron viviendo en el área, que no sufrían defectos congénitos y fueron sometidos a evaluaciones antropométricas y psicológicas. La información fue obtenida por personal rigurosamente entrenado y para el análisis se utilizó el Programa estadístico Stata. La edad media fue 8,78 años. Se realizaron cinco medidas de cociente intelectual en cada niño y todas fueron estadísticamente inferiores a las cifras de zonas urbanas en China. El CI de capacidad cognitiva fue en promedio 89,48 y el índice de comprensión verbal 87,83 . En conclusión, los resultados sugieren que la malnutrición prenatal (IUGR) y postnatal (retardo en crecimiento y bajo peso) afectan la función intelectual de niños escolares. Estos resultados subrayan la importancia de estrategias nutricionales en gestantes y niños pequeños en países en vías de desarrollo ${ }^{(22)}$.

Finalmente, en la actualizada revisión del IUGR por Kesavan y Devaskar ${ }^{(18)}$, en el 2019 se afirma lo siguiente en relación con el desarrollo neurológico:

1. Los infantes con IUGR tienen un alto riesgo de alteraciones en el desarrollo neurológico, incluyendo retardo motor y defectos cognitivos.

2. Un tercio de adultos con IUGR al nacer tuvieron un bajo cociente intelectual.

3. Un pobre ambiente intrauterino aumenta el estrés fetal con mayor producción de glucocorticoides, afectando el desarrollo cerebral.

4. Los estudios en animales han demostrado que las neuronas disminuyen en número y volumen en el hipocampo y en el cerebelo.

5. Las lesiones a los ganglios basales producen dificultades en funciones ejecutivas, mientras que el daño a la corteza fronto-parietal y a sus tractos de sustancia blanca, causan problemas con altos niveles de conocimiento.

6. La alteración cognitiva está asociada de manera independientemente con bajo peso al nacer, talla disminuida y circunferencia cefálica.

7. Los infantes con IUGR pueden presentar pobre rendimiento escolar, debilidad muscular y una capacidad laboral disminuida. El tratamiento con hormona de crecimiento favorece el crecimiento cefálico y puede mejorar el coeficiente intelectual (IQ) en esos pacientes ${ }^{(18)}$.

\section{EN RESUMEN}

- Tener presente la teoría del origen fetal de ciertas enfermedades adultas ${ }^{(1)}$. La programación fetal puede estar relacionada a una desnutrición materna que altera el genoma fetal y origina problemas permanentes de salud (3). La experimentación animal sustenta el concepto de programación fetal ${ }^{(4)}$.

- La desnutrición materna e infantil produce millones de muertes en el mundo, constituyendo el principal problema de salud pública para países en vías de desarrollo ${ }^{(5)}$. La desnutrición materna afecta el desarrollo placentario fetal ${ }^{(10)}$.

- Las intervenciones nutricionales deben centrarse en los primeros dos años de la vida del niño. La alimentación pre-escolar y escolar no han sido muy efectivas ${ }^{(6,7)}$.

- Los micronutrientes para gestantes deben incluir: hierro, folatos, iodo, zinc y vitamina $\mathrm{A}^{(11-13)}$.

- En el desarrollo fetal como origen de la salud y la enfermedad, se hace cada vez más evidente la necesidad de intervención temprana para evitar las consecuencias a corto y largo plazo ${ }^{(14-16)}$.

- La malnutrición fetal origina retardo del crecimiento intrauterino, cuyo conocimiento es impostergable ${ }^{(17,18)}$. La malnutrición fetal altera el desarrollo neurológico ${ }^{(18-22)}$.

\section{LA SITUACIÓN EN EL PERÚ}

Después de una exhaustiva búsqueda, incluyendo desde luego referencias nacionales, no hemos podido encontrar estudios o intervenciones sobre malnutrición materno-fetal, compatibles con la información y conceptos detallados en la presente revisión.

La problemática de salud pública parece estar centrada en la llamada "lucha contra la anemia" que aparece con suma frecuencia en declaraciones, entrevistas y comunicados del Ministerio de Salud, instituciones y prensa nacional.

Las encuestas demográficas y de salud familiar nos anuncian la disminución de la desnutrición infantil, especialmente en lo concerniente a retardo en el crecimiento en niños $<5$ años, con cifras en el 2017 de $12,9 \%$, acompañada por $8 \%$ de obesidad ${ }^{(23)}$. En 2018 , la cifra para desnutrición fue $12,2 \%$, y la de obesidad se incrementó a $8,6 \%$. La cifra de desnutrición de la 
OPS/OMS, para el Perú en el 2018 fue 13,1\%, lo que nos coloca en el puesto 12 de 21 países latinoamericanos ${ }^{(24)}$.

En cuanto a la anemia, fue interesante encontrar la reciente publicación de un exministro de salud titulada "Aprender de la experiencia para vencer la anemia" (25). En ella, se discuten 28 años de intervenciones contra dos flagelos de la salud pública, la desnutrición y la anemia infantil. En un principio, se trataba de cumplir "los objetivos del milenio" de 1990, para disminuir desnutrición infantil ( $<5$ años) 50\%. Al 2015, Perú bajó de 36,5 a 14,6\%. En 1990, el gobierno encontró $68,7 \%$ de anemia pero la hipoxia tuvo que ser manejada con estándares científicos internacionales; en 1996 la cifra fue $60,9 \%$; y en 2015 disminuyó a 46,8\%.

Aparentemente todo iba mejorando, pero el PBI per cápita no se tomó en cuenta ${ }^{(26)}$; sucedió que entre 2005 y 2011, nuestro PBI per cápita fue en promedio 6,46 gracias a la minería y a la agro-exportación, mientras que entre 1990 y 2004 , solo fue 1,5 ; en otras palabras, al disminuir la pobreza, la desnutrición y la anemia mejoraron. Con el estancamiento de nuestra economía a partir del 2014, nuestro PBI fue en promedio 2,1 hasta el 2018, la desnutrición se estabilizó en 13,1\% (PAHO/OMS) y la anemia en 43,6\%. Estos últimos valores, sin embargo, son globales; las cifras para el área rural (21\% de nuestra población) el 2018, fueron $25,7 \%$ y $50,9 \%$ para desnutrición y anemia, respectivamente, lo que subraya un severo problema de salud pública en nuestro país.

\section{Consideraciones finales}

El Perú es un país en vías de desarrollo que pertenece al grupo de ingresos bajos a medianos. Si bien la pobreza global ocurre en el $20,5 \%$ de la población, afecta al $42 \%$ del sector rural ${ }^{(27)}$. El tratamiento de la pobreza está unido al crecimiento económico, pero éste se ha reducido notablemente en la última década. La desnutrición altera la inteligencia al interferir con el nivel de energía, desarrollo motor y la salud general del niño. La pobreza y los bajos niveles educativos de las madres exacerban estos efectos negativos, razón por la cual, pensamos que la desnutrición maternofetal es el principal -y urgente- problema sanitario del Perú.

El empobrecido capital humano nacional muy probablemente adolece de baja talla, bajo índice de masa corporal, pobre conocimiento, falta de educación, disminuida capacidad de trabajo y por ende bajos ingresos, deficiente reproducción y enfermedades crónicas como diabetes, enfermedad cardiovascular, etcétera. Estamos convencidos que este empobrecido capital humano es originado en una importante proporción por la desnutrición de la gestante, que produce serias y frecuentes alteraciones en el genoma del embrión y en el sistema nervioso fetal.

La intervención nutricional precoz es fundamental para manejar adecuadamente el gran problema de la IUGR en nuestro medio. La gran incidencia en Asia y África ofrece importante experiencia en muchos campos, como una agresiva intervención nutricional en los primeros dos años de vida, especial atención en agua y saneamiento y esmerada provisión de micronutrientes ${ }^{(28)}$.

El coeficiente de inteligencia infantil es importante para una vida exitosa, para producir liderazgos y mejores logros escolares. Una fuerte inversión es necesaria no solo para la presente sino para la siguiente generación. No habrá nada más urgente que educar selectivamente a las mujeres que aún sufren condiciones de pobreza, darles asistencia social por especialistas comunitarios, y proveerles de nutrición y suplementos que disminuya la aparición del IUGR en sus niños. Si ello, a pesar de todas las medidas preventivas ocurriese, queda la intervención nutricional precoz, lactancia materna fortificada y micronutrientes. Nuestro capital humano florecerá y ocho años después podremos probar que tendrá un futuro promisor.

Debido a la devastación sanitaria y económica causada por el coronavirus, se ha calculado el incremento de la pobreza a 30\% para fines del 2020 . La directa relación de la malnutrición materno-fetal con la pobreza hace aún más urgente la presente discusión y el desarrollo prioritario de un Plan Nacional de Prevención de la Salud Materno-Infantil.

\section{Correspondencia:}

Luis Caravedo Reyes

Correo electrónico: luis.caravedo@upch.pe

\section{REFERENCIAS BIBLIOGRÁFICAS}

1. Barker D, Winter P, Osmond C, Margetts B, Simmonds S. Weight in infancy and death from ischemic heart disease. Lancet. 1989; 2(8663):57780. doi: 10.1016/s0140-6736(89)90710-1

2. de Onis M, Blössner M, Villar J. Levels and patterns of intrauterine growth retardation in developing 
countries. Eur J Clin Nutr. 1998; 52 Suppl 1:S5-15.

3. Wu G, Bazer F, Cudd T, Meininger C, Spencer T. Maternal nutrition and fetal development. J Nutr. 2004; 134(9):2169-72. doi: 10.1093/jn/134.9.2169

4. Lucas A. Long term programming effects of early nutrition - Implications for the preterm infant. J Perinatol. 2005; 25:S2-S6.

5. Black RE, Allen L, Bhutta Z, et al. Maternal and child undernutrition: Global and regional exposures and health consequences. Lancet. 2008; 371(9608):24360. doi: 10.1016/S0140-6736(07)61690-0

6. Victora CG, Adair L, Fall C, Hallal PC, et al Maternal and child undernutrition: Consequences for adult health and human capital. Lancet. 2008; 371:340357.

7. Bhutta ZA. What works? Interventions for maternal and child undernutrition and survival. Lancet. 2008; 371:417-440.

8. Bryce J. Maternal undernutrition: Efective action at national level. Lancet 2008; 371:510-526.

9. Morris SM. Effective international action against undernutrition: Why has it proven so difficult and what can be done to accelerate progress? Lancet 2008; 371:608-621.

10. Belkacemi L. Maternal undernutrition influences placental-fetal development. Biol Reprod. 2010; 83:325-331.

11. Ahmed T. Global burden of maternal and child undernutrition and micronutrient deficiencies. Ann Nutr Metab. 2012; 61(suppl 1):8-17.

12. Cetin I, Laoreti A. The importance of maternal nutrition for health. J pediatric neonatal individ medicine. 2015; 4(2):1-11,

13. Morrison JL, Regnault TRH. Nutrition in pregnancy: Optimising maternal diet and fetal adaptations to altered nutrient supply. Nutrients 2016; 8(6):342.

14. Fall CHD. Fetal malnutrition and long-term outcomes. Nestle Nutr Inst Workshop Ser .2013; 74:11-25.

15 . Wang N. The famine exposure in early life and metabolic syndrome in adulthood. Clinical Nutrition. 2017; 36:253-259.

16. Mandy M, Nyirenda M. Developmental Origings of Health and Disease: The relevance to developing nations. Int Health. 2018; 10:66-70.

17. Sharma D. Intrauterine growth restriction: Antenatal y postnatal aspects. Pediatrics. 2016; 10:67-83.
18. Kesavan K, Devaskar SU. Intrautero growth restriction: Postnatal monitoring and outcomes. Pediatr Clin N Am. 2019; 66:403-423.

19. Lucas A, Morley R, Cole TJ. Randomised trial of early diet in preterm babies and later intelligence quotient. BMJ. 1998; 317:1481-1487.

20. Isaacs EB, Morley R, Lucas A. Early diet and general cognitive outcome at adolescence in children born at or below 30 weeks gestation. J Pediatr. 2009; 155(2):229-234.

21. Keunen K. Impact of nutrition on brain development and its neuroprotective implications following preterm birth. Pediatr Res. 2015; 77(1-2):148-55. doi: $10.1038 /$ pr.2014.171

22. Li C. Effect of prenatal and postnatal malnutrition on intellectual functioning in early school-aged children in rural western China. Medicine (Baltimore). 2016; 95(31):e4161.

23. Instituto Nacional de Estadística e Informática. Encuestas demográficas y de salud familiar. Lima: Instituto Nacional de Estadística e Informática; 2019.

24. Uceda J. Reforma del Sistema de Salud en el Perú. Lima Blog de Jorge Uceda; 2020. (Citado el 20 de agosto del 2020) Disponible en: http://jorgeuceda. blogspot.com/2020/04/

25. Ugarte O. Aprender de la experiencia para vencer la anemia. Ideele. 2019; 284:0-0. (Citado el 20 de agosto del 2020) Disponible en: https://revistaideele. com/ideele/content/aprender-de-la-experiencia-paravencer-la-anemia

26. Instituto Nacional de Estadísticas e Informática. Panorama de la economía peruana 1950-2018. Lima: Instituto Nacional de Estadística e Informática; 2019.

27. Instituto Nacional de Estadísticas e Informática. Pobreza en el Perú. Lima: Instituto Nacional de Estadística e informática; 2018.

28. Lahariya C. Maternal and child undernutrition: The Lancet Series and Indian perspective. Indian Pediatrics. 2008; 45:298-299.

Recibido: 29/08/2020

Aceptado: 14/12/2020 